
\title{
$\begin{array}{ll}\text { Research Square } & \begin{array}{l}\text { Preprints are preliminary reports that have not undergone peer review. } \\ \text { They should not be considered conclusive, used to inform clinical practice, } \\ \text { or referenced by the media as validated information. }\end{array}\end{array}$ \\ Twice vitrification-warming procedures has no effect on frozen-thawed embryo transfer outcomes
}

\section{xiaoyue Shen}

Nanjing Drum Tower Hospital: Nanjing University Medical School Affiliated Nanjing Drum Tower Hospital https://orcid.org/0000-0001-9525-6137

\section{Min Ding}

Nanjing Drum Tower Hospital: Nanjing University Medical School Affiliated Nanjing Drum Tower Hospital

\section{Yuan Yan}

Nanjing Drum Tower Hospital: Nanjing University Medical School Affiliated Nanjing Drum Tower Hospital

\section{Shanshan Wang}

Nanjing Drum Tower Hospital: Nanjing University Medical School Affiliated Nanjing Drum Tower Hospital jianjun Zhou ( $\sim$ zhou6jj@hotmail.com )

Drum Tower Hospital Affiliated to Nanjing University Medical school

Jun Xing

Nanjing Drum Tower Hospital: Nanjing University Medical School Affiliated Nanjing Drum Tower Hospital

\section{Research}

Keywords: frozen-thawed embryo transfer, repeated cryopreservation, embryo, pregnancy outcomes, macrosomia

Posted Date: November 15th, 2021

DOI: https://doi.org/10.21203/rs.3.rs-1059310/v1

License: (9) (1) This work is licensed under a Creative Commons Attribution 4.0 International License. Read Full License 


\section{Abstract \\ Background}

To evaluate the frozen-thawed embryo transfer (FET) outcomes of repeated cryopreservation by vitrification of blastocysts derived from vitrified-warmed day3 embryos in patients who experienced implantation failure previously.

\section{Methods}

We retrospect the files of patients who underwent single frozen-thawed blastocyst transfer cycles in our reproductive medical center from January 2013 to December 2019. 127 patients transfer of vitrified-warmed blastocysts derived from vitrified-warmed day3 embryos were defined as twice-cryopreserved group. 1567 patients who transfer blastocysts that had experienced once vitrified-warmed were used as once-cryopreserved group. None of them was pregnant at the previous FET. The outcomes were compared between two groups after a 1:1 propensity score matching (PSM).

\section{Results}

The clinical pregnancy rate was $52.76 \%$, live birth rate was $43.31 \%$ in twice-cryopreserved group. After PSM,108 pairs of patients were generated for comparison. The clinical pregnancy rate, live birth rate or miscarriage rate was not significantly different between two groups. Logistic regression analysis indicated that double vitrification-warming procedures did not affect FET outcomes in terms of clinical pregnancy rate (OR 0.83 , $95 \% \mathrm{Cl} 0.47-1.42)$, live birth rate (OR $0.93,95 \% \mathrm{Cl} 0.54-1.59)$, miscarriage rate (OR $0.7295 \% \mathrm{Cl} 0.28-1.85$ ). Furthermore, the pregnancy complications rate, gestational age or neonatal abnormalities rate between two groups was also comparable, while twice vitrification-warming procedures might increase the macrosomia rate $(19.6 \%$ vs. $6.3 \%, P=0.05)$.

\section{Conclusion}

Transfer of double vitrified-warmed embryo at cleavage stage and subsequent blastocyst stage did not affect live birth rate and neonatal abnormalities rate, but there was a tendency to increase macrosomia rate, which needs further investigation.

\section{Background}

It has been decades since the first IVF baby born, and then assisted reproduction has acquired significant advances, the number of available embryos in each IVF cycle has also increased, resulting in a slew of surplus embryo cryopreservation. Cryopreservation of embryos will increase the cumulative pregnancy rate for a single cycle of ovarian stimulation, the clinical pregnancy rate and live birth rate was similar with fresh embryo transfer, while frozen-thawed embryo transfer (FET) decreases the risk of ovarian hyperstimulation syndrome (OHSS), provides optimal endometrial preparations in case of premature progesterone rises, adenomyosis or 
hydrosalpinx [1-3]. Embryo cryopreservation has become a widespread reliable procedure in assisted reproductive technologies [4]. However, cryopreservation procedure may be have negative effects on both mothers and babies when compared with fresh embryo transfer $\square$ such as higher rate of hypertensive disorders of pregnancy, large for gestational age (LGA) and higher birthweight [5-7].

Single embryo transfer was global recommended to reduce multiple pregnancies[8]. In case of multiple embryos were frozen in one cryo-straw previously, these vitrified embryos will be warmed together, when only one embryo were transferred, the other warmed embryos have to be vitrified again, so these embryos would undergo twice vitrification-warming procedures. Some studies have focused on whether repeated cryopreservation process affect the pregnancy and perinatal outcomes. One study showed that twice-frozenthawed embryos have a lower post-thaw survival rate but equivalent pregnancy outcomes to once-frozen embryos[9]. Others found repeated cryopreservation process impairs embryo implantation potential, with lower birth rate and higher miscarriage rate[10,11]. However, the patients underwent repeated cryopreserved embryo transfer may be always experienced previous embryo transfer failure, so the control group should be consistent with this, which may the sample selection bias in these studies. And the most common situation in our center was that two embryos were frozen in one cryo-straw at cleavage stage, after single embryo transfer, the surplus thawed embryos will culture to blastocyst stage and vitrified again, so these embryos underwent twice vitrification-warming procedures at cleavage stage and subsequent blastocyst stage, which was different from some other studies when the repeated cryopreservation process was performed at the same stage.

In this retrospective study, we investigated the effectiveness and safety of twice vitrification-warming procedures at cleavage stage and subsequent blastocyst stage in in patients who experienced previous failure. The pregnancy and perinatal outcomes were compared between the twice-cryopreserved group and the oncecryopreserved group.

\section{Patients And Methods}

\section{Patients}

The medical files of all frozen-thawed blastocyst cycles from January 2013 to December 2019 at Reproductive Medicine Center of Nanjing Drum Tower Hospital were retrospectively reviewed. We established inclusion and exclusion criteria. Inclusion criteria $\mathbb{X}(1)$ single blastocyst transfer, (2) the patient had experienced at least one frozen-thawed cycle and had not received a live birth in the precede cycle. Exclusion criteria $\mathbb{( 1 )}$ patients received multiple births, (2) endometriosis, (3) preimplantation genetic testing cycles, (4) donor cycles, (5) history of recurrent pregnancy loss, and (6) uterine pathology. Totally 1694 women patients were included in this study. They were divided into two groups based on times of embryos had been cryopreserved冈oncecryopreserved group $₫ n=1567 \rrbracket$ and twice-cryopreserved group $\ n=127 \rrbracket$. Among twice-cryopreserved group, 127 FET cycles were those patients in whom two embryos were frozen by vitrification in one cryo-straw at cleavage stage, after single embryo transfer, the surplus embryo will culture to blastocyst stage in vitro, then refrozen by vitrification, then transferred after rethawing.

\section{IVF/ICSI procedure and embryos culture}


Depending on the patients' age, ovarian reserve function, and ovarian responses in the previous ovulation cycle, gonadotrophin-releasing hormone agonist or antagonist protocols were used. When two to three dominant follicles reached a diameter of $18 \mathrm{~mm}$, human chorionic gonadotropin (HCG) was injected. The oocytes were retrieved under transvaginal ultrasound guidance 36-38 hours after HCG administration. Retrieved oocytes were then fertilized in conventional IVF or intracytoplasmic sperm injection (ICSI). Two pronuclei and a second polar body were examined for normal fertilization at 16-18 h post-insemination. Embryos were cultured in $\mathrm{G} 1 / \mathrm{G} 2$ sequential media (Vitrolife, Goteborg, Sweden) at $37^{\circ} \mathrm{C}$ in a 6\% CO2, 5\% 02 and 89\% N2 high-humidity incubator. Blastocyst morphological evaluation was based on the Gardner scoring system [12].

\section{Vitrification cryopreservation and thawing procedures}

The vitrification cryopreservation and thawing procedures was performed in embryos $₫$ vitrification kit was produced and available at Kitazato Co., Fujinomiya, Japan. Before vitrification, the blastocyst was artificially shrunken by laser drilling.

Cleavage embryos or shrunken blastocysts were equilibrated in equilibration solution for 5-8 min at room temperature, then placed into vitrification solution, 1 minute later the embryos were loaded onto the cryotop strip and plunged into liquid nitrogen immediately. For warming, the cryotop strip was removed from the liquid nitrogen and immediately transferred to thawing solution for 1 minute at $37 \circ \mathrm{C}$, dilution solution for 3 minutes, and then washed twice in washing solution 1 and washing solution 2 for 5 min each. After warming, embryos were cultured in embryo culture medium at $37^{\circ} \mathrm{C}$ in $6 \% \mathrm{CO} 2$ for $2-4 \mathrm{~h}$ until transfer.

\section{Outcome Measures}

Baseline demographics for each patient included age, BMI, duration of infertility, parity, type of infertility and cause of infertility, insemination methods (IVF or ICSI), endometrial thickness \and endometrium preparation protocol for FET.

Serum HCG was measured after embryo transfer 12-14 days. Clinical pregnancy was defined as the presence of gestational sacs observed on an ultrasound scan at least 5 weeks after the embryo transfer. The fetal loss before 28 weeks was defined as miscarriage. The embryo was implanted outside the uterine cavity called ectopic pregnancy. Such as hypertensive disorders of pregnancy, gestational diabetes mellitus, intrahepatic cholestasis during pregnancy and placenta previa were included in pregnancy complications. Any singleton birth $\geq 28$ weeks of gestation was considered a live singleton birth. The neonatal outcomes evaluated were

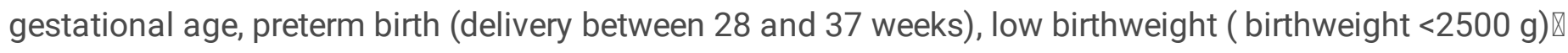
macrosomia ( birthweight $\geq 4000 \mathrm{~g}$ ) $₫$ gender and neonatal abnormality.

\section{Statistical analysis}

Means \pm SD for continuous variables $₫$ percentages for categorical variables were calculated. The data were analyzed using the chi-squared test or Student's t-test. P-values $<0.05$ was considered statistically significant. 1:1 Propensity score matching was used in data analysis to eliminate the influence of baseline characteristics on outcomes. The differential variables include female age at retrieval, average embryo transfer cycles, parity, basal FSH, type of infertility and cause of infertility. To analyze the associations between the cryopreserved times and pregnancy outcomes, logistic regression models were conducted for each outcome indicator using 
the after-matching data, and odds ratio (OR) and its $95 \% \mathrm{Cl}$ before and after adjusting for confounders were calculated. Statistical analysis was performed using SPSS statistical package version 26.0.

\section{Results}

\section{Clinical characteristics and outcomes of the twice-cryopreserved grou $p$}

Patients' overall baseline clinical demographics of the twice-cryopreserved group were displayed in Table 1. The female age at retrieval or embryo transfer were $29.61 \pm 3.96$ and $34.28 \pm 4.12$ years. The average BMI and basal FSH of the study population were $22.33 \pm 2.89 \mathrm{~kg} / \mathrm{m}^{2}$ and $6.7 \pm 1.77 \mathrm{mlU} / \mathrm{mL}$, respectively. In the FET cycles, the hormone-replacement therapy (HRT) predominated in endometrium preparation (104 cycles). In the 127 blastocysts, ninety-one were day 5 blastocysts, while the remaining 36 were day 6 blastocysts. 
Table 1

Overall demographics,baseline IVF characteristics of the twice-cryopreserved group $(n=127)$.

\begin{tabular}{|c|c|}
\hline \multicolumn{2}{|l|}{ Demographics } \\
\hline Female age at retrieval ,years & $29.61 \pm 3.96$ \\
\hline Female age at embryo transfer,years & $34.28 \pm 4.12$ \\
\hline Parity & $0.78 \pm 0.47$ \\
\hline Body mass index,kg/m² & $22.33 \pm 2.89$ \\
\hline Basal FSH, mlU/mL & $6.70 \pm 1.77$ \\
\hline Duration of infertility ,years & $4.09 \pm 2.65$ \\
\hline Endometrial thickness, mm & $9.32 \pm 1.68$ \\
\hline \multicolumn{2}{|l|}{ Type of Infertility, n } \\
\hline Primary & 71 \\
\hline Secondary & 56 \\
\hline \multicolumn{2}{|l|}{ Cause of Infertility, $n$} \\
\hline Ovulation disorder & 6 \\
\hline Tubal factor & 78 \\
\hline Male factors & 11 \\
\hline Other factors & 32 \\
\hline \multicolumn{2}{|l|}{ IVF characteristics } \\
\hline \multicolumn{2}{|l|}{ Insemination method, $\mathrm{n}$} \\
\hline IVF & 96 \\
\hline ICSI & 31 \\
\hline Average number of embryo transfer cycle & $3.65 \pm 1.12$ \\
\hline Endometrial thickness, mm & $9.32 \pm 1.68$ \\
\hline \multicolumn{2}{|l|}{ Endometrial preparation protocol, $\mathrm{n}$} \\
\hline HRT & 104 \\
\hline Ovulation induction & 13 \\
\hline Modified Natural Cycle & 10 \\
\hline \multicolumn{2}{|l|}{ Day of embryo cryopreservation, $n$} \\
\hline D5 & 91 \\
\hline
\end{tabular}




\begin{tabular}{|lc|}
\hline Demographics \\
\hline D6 & 36 \\
\hline Embryo level, n104 \\
\hline High quality embryos \\
\hline Good quality embryos & 76 \\
\hline
\end{tabular}

Clinical outcomes and perinatal outcomes of the study population were listed in Table 2. The clinical pregnancy rate and live birth rate were $52.76 \%$ and $43.31 \%$. In all clinical pregnant people, $14.93 \%$ patients occurred miscarriage before 12 weeks, 11 pregnant women had pregnancy complications and no ectopic pregnancy occurred. According to the follow-up results, 55 live singleton deliveries were obtained, including 28 male babies and 23 female babies. The average gestational age was $37.65 \pm 2.21$ weeks, and the average birthweight was $3412.60 \pm 666.37 \mathrm{~g}$. The preterm birth in singletons was $16.36 \%$, and rate of low birthweight and macrosomia were $7.27 \%$ and $16.36 \%$, respectively. There was no neonatal disease and malformation occurred. 
Table 2

Clinical outcomes and perinatal outcomes of twicecryopreserved embryos transferred $(n=127)$.

\begin{tabular}{|ll|}
\hline Clinical Outcomes & \\
\hline Biochemical pregnancy rate (\%) & $72 / 127(56.69)$ \\
\hline Clinical pregnancy rate (\%) & $67 / 127(52.76)$ \\
\hline Miscarriage rate (\%) & $11 / 67(16.42)$ \\
\hline Early (<12 weeks) & $10 / 67(14.93)$ \\
\hline Late (12-28 weeks) & $1 / 67(1.49)$ \\
\hline Ectopic pregnancy & $0 / 67(0)$ \\
\hline Pregnancy complication (\%) & $11 / 67(16.42)$ \\
\hline Live birth rate (\%) & $55 / 127(43.31)$ \\
\hline Perinatal outcomes & \\
\hline Singleton delivery & 55 \\
\hline Gestational age,weeks & $37.65 \pm 2.21$ \\
\hline Preterm birth rate (<37 weeks) (\%) & $9 / 55(16.36)$ \\
\hline Full-term birth rate (37-42 weeks) (\%) & $46 / 55(83.63)$ \\
\hline Gender & \\
\hline Boys & 28 \\
\hline Girls & 27 \\
\hline Neonatal abnormalities (\%) & $0 / 55(0)$ \\
\hline Birthweight ,g & $3412.60 \pm 666.37$ \\
\hline Low birthweight (<2500 g) (\%) & $4 / 55(7.27)$ \\
\hline Macrosomia ( $\geq 4000 \mathrm{~g})(\%)$ & $9 / 55(16.36)$ \\
\hline
\end{tabular}

The propensity score matching of basic characteristics between once-cryopreserved group and twicecryopreserved group

Among a total of 1694 embryos, 127 were re-thawed and underwent transfer (twice-cryopreserved group), 1567 were underwent a single thawed embryos transfer (once-cryopreserved group). The comparison of clinical characteristics between the two groups were listed in Table 3. The female patients were younger $(29.61 \pm 3.96$ vs. $31.42 \pm 4.96, p<0.001)$, basal FSH was lower $(6.70 \pm 1.77$ vs. $7.53 \pm 3.19, p<0.001)$ in twice-cryopreserved group. More embryo transfer cycles were performed in the twice-cryopreserved group (mean cycles:3.65) than in once-cryopreserved group (mean cycles: 2.94$)(P<0.001)$ and the parity was more in the twice-cryopreserved 
group $(0.78 \pm 0.47$ vs. $0.32 \pm 0.49, \mathrm{P}<0.001)$. The distribution of infertility type, cause of infertility was different between the two groups.

Table 3 The propensity score matching of basic characteristics between once-cryopreserved group and twicecryopreserved group.

In order to eliminate the influence of baseline characteristics on outcomes, a 1:1 PSM was performed between the two groups. Finally, a total of 108 pairs were matched by their propensity score. Before propensity score matching, two groups had significantly different maternal and IVF characteristics but the propensity score matching balanced these characteristics between the cohorts (Table 3), suggesting the matched cohorts had highly similar baseline characteristics.

\section{Pregnancy outcomes between the once-cryopreserved group and twice-cryopreserved group after matching}

The clinical outcomes were summarized in Table 4 . The clinical pregnancy rate $(51.85 \%$ vs. $57.41 \%, p=0.41)$, live birth rate ( $44.44 \%$ vs. $42.59 \%, p=0.78)$ and miscarriage rate $(16.07 \%$ vs. $20.97 \%, p=1.00)$ were not significantly different between the two groups. No late miscarriage and ectopic pregnancy occurred in both groups. There were 5 patients with pregnancy complications in once-cryopreserved group, and 7 patients with pregnancy complications in twice-cryopreserved group. There was no statistical difference in the incidence of pregnancy complications ( $12.50 \%$ vs. $8.06 \%$ ) between the two groups. 
Before matching

\begin{tabular}{|c|c|c|c|c|}
\hline $\begin{array}{l}\text { Once- } \\
\text { cryopreserved } \\
\text { group }\end{array}$ & $\begin{array}{l}\text { Twice- } \\
\text { cryopreserved } \\
\text { group }\end{array}$ & $\begin{array}{l}\text { P- } \\
\text { value }\end{array}$ & $\begin{array}{l}\text { Once- } \\
\text { cryopreserved } \\
\text { group }\end{array}$ & $\begin{array}{l}\text { Twice- } \\
\text { cryopreserved } \\
\text { group }\end{array}$ \\
\hline
\end{tabular}

No. of cycles

Female age at retrieval『years

Average embryo

transfer cycles

Parity

1567

$31.42 \pm 4.96$

127

$29.61 \pm 3.96$

$2.94 \pm 1.08$

$3.65 \pm 1.12$

$0.32 \pm 0.49$

$0.78 \pm 0.47$

$22.33 \pm 2.89$

Body mass index, $\quad 22.64 \pm 3.27$

$\mathrm{kg} / \mathrm{m}^{2}$

Basal FSH ,mIU/mL

Duration

of infertility, years

Endometrial

thickness,mm

Insemination

method

IVF

ICSI

Type of Infertility

Primary

Secondary

Day of embryo cryopreservation

$7.53 \pm 3.19$

$3.95 \pm 3.16$

$9.45 \pm 1.69$

$9.32 \pm 1.68$

$6.70 \pm 1.77$

$4.09 \pm 2.65$

0.61

0.38

$9.26 \pm 1.31$

108

$<0.001$

$29.94 \pm 4.86$

$<0.001 \quad 3.28 \pm 1.35$

$<0.001 \quad 0.69 \pm 0.54$

$0.75 \pm 0.50 \quad 0.36$

$0.75 \pm 0.50 \quad 0.36$

$0.25 \quad 22.43 \pm 2.90$

$22.39 \pm 3.05$

0.93

0.90

$4.13 \pm 2.97$

$4.28 \pm 2.70$

0.69

$6.74 \pm 1.78 \quad 0.34$

$<0.001 \quad 7.00 \pm 2.31$

$29.88 \pm 4.05 \quad 0.93$

$3.58 \pm 1.06 \quad 0.07$

711

856

96

76

80

390

31

32

28

71

0.02

0.89

56

57

51

58
D5

1053

514

D6

514

91

36

Endometrial

preparation

protocol

HRT

1269

104

86

11

Ovulation

148

13

10

Natural Cycle

150

(NC)

Cause of

$<0.001$

0.25 
Infertility

\begin{tabular}{|c|c|c|c|c|c|}
\hline $\begin{array}{l}\text { Ovulation } \\
\text { disorder }\end{array}$ & 73 & & 1 & 4 & \\
\hline Tubal factor & 648 & 78 & 58 & 65 & \\
\hline Male factors & 166 & 11 & 16 & 11 & \\
\hline Other factors & 680 & 32 & 33 & 28 & \\
\hline Embryo quality & \multicolumn{4}{|c|}{0.25} & 0.34 \\
\hline $\begin{array}{l}\text { High quality } \\
\text { embryos }\end{array}$ & 947 & 76 & 56 & 64 & \\
\hline \multirow{12}{*}{$\begin{array}{l}\text { Good quality } \\
\text { embryos }\end{array}$} & 620 & 51 & 52 & 44 & \\
\hline & \multicolumn{4}{|c|}{$\begin{array}{l}\text { Table } 4 \text { The clinical outcomes between once-cryopreserved group } \\
\text { and twice-cryopreserved group after propensity score matching. }\end{array}$} & \\
\hline & & $\begin{array}{l}\text { Once- } \\
\text { cryopreserved } \\
\text { group }\end{array}$ & $\begin{array}{l}\text { Twice- } \\
\text { cryopreserved } \\
\text { group }\end{array}$ & P-value & \\
\hline & No. of cycles & 108 & 108 & - & \\
\hline & $\begin{array}{l}\text { Biochemical } \\
\text { pregnancy } \\
\text { rate }(\%)\end{array}$ & 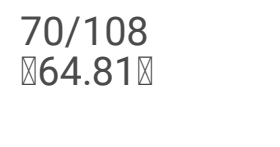 & $\begin{array}{l}61 / 108 \\
\otimes 56.48 \rrbracket\end{array}$ & 0.21 & \\
\hline & $\begin{array}{l}\text { Clinical } \\
\text { pregnancy }(\%)\end{array}$ & 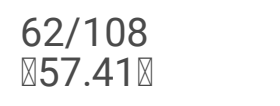 & $\begin{array}{l}56 / 108 \\
\square 51.85 \square\end{array}$ & 0.41 & \\
\hline & Miscarriage (\%) & $13 / 62$ (20.97) & 9/56 (16.07) & 1.00 & \\
\hline & $\begin{array}{l}\text { Early }(<12 \\
\text { weeks) }\end{array}$ & $\begin{array}{l}13 / 62 \\
(20.97 \%)\end{array}$ & 9/56 (16.07) & & \\
\hline & $\begin{array}{l}\text { Late }(12-28 \\
\text { weeks) }\end{array}$ & $0 / 62(0.0)$ & $0 / 56(0.0)$ & & \\
\hline & $\begin{array}{l}\text { Ectopic } \\
\text { pregnancy (\%) }\end{array}$ & $0 / 62(0.0)$ & $0 / 56(0.0)$ & 1.00 & \\
\hline & $\begin{array}{l}\text { Live birth } \\
\text { rate }(\%)\end{array}$ & $\begin{array}{l}48 / 108 \\
(44.44)\end{array}$ & $\begin{array}{l}46 / 108 \\
(42.59)\end{array}$ & 0.78 & \\
\hline & $\begin{array}{l}\text { Pregnancy } \\
\text { complication } \\
(\%)\end{array}$ & 5/62ه8.06》 & 7/56ه12.50区 & 0.43 & \\
\hline
\end{tabular}

Moreover, we used regression model to adjust factors that might affect the results according to female retrieval age, BMI, FSH, embryo transfer cycles, embryo quality. The adjusted outcome showed that the clinical pregnancy rate (AOR $0.83,95 \% \mathrm{Cl} 0.47,1.42)$, live birth rate (AOR $0.93,95 \% \mathrm{Cl} 0.54,1.81$ ) or early miscarriage rate (AOR $0.72,95 \% \mathrm{Cl} 0.28,1.85$ ) was no statistical difference (Table 5). 
Table 5

Adjusted Clinical pregnancy rate/Live birth rate/Early miscarriage after propensity score matching.

\begin{tabular}{|llllllllll|}
\hline $\begin{array}{l}\text { Embryo } \\
\text { cryopreservation } \\
\text { times }\end{array}$ & \multicolumn{3}{l}{ Clinical pregnancy rate (\%) } & \multicolumn{3}{c|}{ Live birth rate (\%) } & \multicolumn{4}{c|}{$\begin{array}{c}\text { Early spontaneous } \\
\text { abortion(\%) }\end{array}$} \\
\cline { 2 - 10 } & $\%$ & AOR & $\mathbf{9 5 \% C l}$ & $\%$ & AOR & $\mathbf{9 5 \% C l}$ & $\%$ & AOR & $\mathbf{9 5 \% C l}$ \\
\hline Once & 57.41 & 1 & Reference & 44.44 & 1 & Reference & 20.97 & 1 & Reference \\
\hline Twice & 51.85 & 0.83 & $0.47-1.42$ & 42.59 & 0.93 & $0.54-1.59$ & 16.07 & 0.72 & $0.28-1.85$ \\
\hline
\end{tabular}

We adjusted the female retrieval age, BMI, FSH, embryo transfer cycles, parity, the type of infertility and the quality of embryos. AOR is the adjusted odds ratio in logistic regression analysis, representing the risk ratio of clinical pregnancy/live birth/early miscarriage in the twice-cryopreserved group versus once-cryopreserved group. AOR greater than 1 represents increased risk, and AOR less than 1 represents reduced risk. $95 \% \mathrm{Cl}$ is the $95 \%$ confidence interval of AOR.

In addition, we conducted a stratified analysis based on the day of embryo cryopreservation, embryo quality and previous labor history, the difference in live birth rates between the two groups is shown in Table 6. The results showed that transferring D5 or D6 blastocyst,delivery history or not, the live birth rate had no significant difference between the two groups $(P>0.05)$. In both groups, high-quality blastocysts for transferring resulted in a higher live birth rate than good-quality blastocysts $(P<0.01)$. However, there was also no significant difference in the live birth rate on transferring the same level embryos between the two groups.

Table 6

The live birth rate analyzed by stratification between once-cryopreserved group and twice-cryopreserved group after propensity score matching.

\begin{tabular}{|llllll|}
\hline & \multicolumn{2}{c}{ Once-cryopreserved group } & \multicolumn{2}{c|}{ Twice-cryopreserved group } & P-value \\
\cline { 2 - 5 } & Live birth rate (\%) & P-value & Live birth rate (\%) & P-value & \\
\hline Day of embryo cryopreservation & & 0.87 & & 0.26 \\
\hline D5 & $39 / 87(44.83)$ & & $37 / 81(45.68)$ & 0.91 \\
\hline D6 & $9 / 21(42.86)$ & & $9 / 27(33.33)$ & 0.50 \\
\hline Embryo quality & & 0.08 & & $<0.01$ \\
\hline High quality embryos & $30 / 56(53.57)$ & & $35 / 64(54.69)$ & 0.90 \\
\hline Good quality embryos & $18 / 52(34.6)$ & & $11 / 44(25)$ & 0.28 \\
\hline Parity & & 0.11 & & 0.47 \\
\hline No,Primipara & $13 / 38(34.21)$ & & $14 / 29(48.28)$ & 0.24 \\
\hline Yes,Multipara & $35 / 70(50)$ & & $32 / 79(40.51)$ & & 0.24 \\
\hline
\end{tabular}


Of the 216 embryo transfer cycles, there were a total of 94 delivered singletons, including 46 derived from the twice-cryopreserved group and 48 from the once-cryopreserved group. There were no significant differences in the mean gestational age ( $37.85 \pm 2.01$ vs. $37.65 \pm 1.92, P=0.62)$, gender ratio (girls/boys, $23 / 23$ vs. $25 / 23$, $P=0.84)$, mean birthweight ( $3497.02 \pm 642.01$ vs. $3371.98 \pm 522.86, P=0.30)$, macrosomia rate $(19.56 \%$ vs. $6.35 \%$, $\mathrm{P}=0.05)$ and preterm birth rate $(13.04 \%$ vs. $12.5 \%, P=0.94)$ between the two groups (Table 7$)$. A singleton baby born in the once-cryopreserved group had angioma defect and the rate of congenital birth defects was 1/48 in the once-cryopreserved group. While no newborn anomalies were occurred in the twice-cryopreserved group. 
Table 7

Neonatal characteristics of live-born singletons between once-cryopreserved group and twicecryopreserved group after propensity score matching.

\begin{tabular}{|c|c|c|c|}
\hline & Once-cryopreserved group & Twice-cryopreserved group & P-value \\
\hline No. of cycles & 108 & 108 & \\
\hline Live-born singletons & 48 & 46 & \\
\hline Gestational age, weeks & $37.65 \pm 1.92$ & $37.85 \pm 2.01$ & 0.62 \\
\hline Preterm birth (<37 weeks) (\%) & $6 / 48(12.5)$ & $6 / 46(13.04)$ & 0.94 \\
\hline Term birth ( $\geq 37$ weeks) (\%) & $42 / 48(87.5)$ & $40 / 46(86.96)$ & 0.94 \\
\hline Neonatal abnormalities (\%) & $1 / 48(2.08)$ & $0 / 46(0.0)$ & 1.00 \\
\hline Birthweight, g & $3371.98 \pm 522.86$ & $3497.02 \pm 642.01$ & 0.30 \\
\hline Low birthweight (<2500 g) (\%) & $0 / 48(0.0)$ & $0 / 46(0.0)$ & 1.00 \\
\hline Macrosomia ( $\geq 4000 \mathrm{~g}$ ) (\%) & $3 / 48(6.35)$ & 9/46 (19.56) & 0.05 \\
\hline \multicolumn{4}{|l|}{ Gender } \\
\hline Boys & 23 & 23 & \\
\hline Gestational age, weeks & $37.83 \pm 1.34$ & $37.70 \pm 1.96$ & 0.79 \\
\hline Birthweight, g & $3503.48 \pm 384.77$ & $3598.26 \pm 723.09$ & 0.58 \\
\hline Low birthweight (<2500 g) (\%) & $0 / 23(0.0)$ & $0 / 23(0.0)$ & 1.00 \\
\hline Macrosomia ( $\geq 4000 \mathrm{~g}$ ) (\%) & $2 / 23(8.69)$ & $6 / 23(26.09)$ & 0.24 \\
\hline Girls & 25 & 23 & \\
\hline Gestational age, weeks & $37.48 \pm 2.35$ & $38.00 \pm 2.09$ & 0.42 \\
\hline Birthweight, g & $3251.00 \pm 606.50$ & $3395.78 \pm 546.63$ & 0.39 \\
\hline Low birthweight (<2500 g) (\%) & $0 / 25(0.0)$ & $0 / 23(0.0)$ & 1.00 \\
\hline Macrosomia ( $\geq 4000 \mathrm{~g}$ ) (\%) & $1 / 25(4.0)$ & $3 / 23(13.04)$ & 0.26 \\
\hline
\end{tabular}

In order to eliminate the possible effect of infant gender on birthweight, we compared the differences in offspring birth weight and mean gestational weeks between the once-cryopreserved group and the twicecryopreserved group in boys and girls, respectively (Table 7). The results shown that both boys and girls, there was no significant difference in gestational week and the birth weight between the two groups. Whether boys or girls, the macrosomia rate in the twice-cryopreserved group was slightly higher than that in the oncecryopreserved group, but it did not reach statistical difference $(P>0.05)$.

\section{Discussion}


Embryo cryopreservation is a crucial part of assisted reproductive technology, and the proportion of FET had grown remarkably. In order to reduce the risk of multiple pregnancies and pregnancy complications, single embryo transfer is preferred in the case of suitable patient conditions and embryo quality. Studies have found that compared with embryos in cleavage stage, single blastocyst transfer significantly improves clinical pregnancy rate and implantation rate [13-16]. As multiple cleavage stage embryos might be frozen in one cryostraw previously, after single embryo transfer, the surplus thawed embryos will be cultured to blastocyst stage and vitrified again, if this time of embryo transfer failed, these patients might choose to transfer these embryos which underwent twice vitrification-warming procedures. In this study, we found that transfer of twice vitrifiedwarmed embryo at cleavage stage and subsequent blastocyst stage did not affect pregnancy and perinatal outcomes.

There were several case reports demonstrating healthy live birth after a frozen embryo transfer with embryo that were frozen and thawed twice [17-21]. And some early retrospective studies have also shown that the embryos re-cryopreservation does not affect clinical pregnancy rates [9, 22-24]. However, other recent studies have shown that transfer of twice frozen-thawed embryos increase the rate of miscarriage and decreases the clinical pregnancy rate and live birth rate $[10,11,25]$. The discrepancies in the results of these studies may be due to the different methods used to freeze embryos,embryos frozen at different stages and mismatched baseline of clinical characteristics. Furthermore, these studies may exist the sample selection biases, as the patients underwent repeated cryopresered embryo transfer may always experience previous embryo transfer failure, so the control group should be consistent with this. So, the control group in our study were also experienced previous embryo transfer failure. Furthermore, in order to eliminate the influence of clinical baseline characteristics on results, a propensity score and logistic regression was used to control for potential confounding differences in our study.

After PSM, the clinical pregnancy rate, miscarriage rate, live birth rate or the incidence of pregnancy complications had no difference between the once-cryopreserved group and the twice-cryopreserved group. There was a difference in clinical pregnancy and live birth rates between blastocysts developing on Day 5 (D5) and Day 6 (D6),the clinical pregnancy rate and live birth rate were significantly higher following D5 compared to D6 blastocysts [26, 27]. In addition $\square$ the quality of embryos plays a crucial role in embryo implantation and live birth rate[28, 29]. In the present study, we stratified the live birth rates based on the day of embryo cryopreservation, embryo quality between once-cryopreserved group and twice-cryopreserved group and found no significant difference. Logistic regression after adjusting for relevant confounding factors showed that the number of vitrification-warming procedure was not related to the clinical pregnancy rate, live birth rate or early miscarriage rate, which was not consist with other studies. Zheng et al. (2017) reported the miscarriage rate of the twice-cryopreserved group was higher and the live birth rate was lower than control group, which may due to two cryopreservation methods were used in their study, slow freezing of cleavage embryos followed by vitrification of blastocysts[10]. In our study, we only used one cryopreservation method, vitrification. Compared with slow-freezing method, embryo vitrification is an ultra-rapid cryopreservation method which prevent ice formation, reduce embryo cryoinjury, have higher embryo survival rate and live birth rate [30, 31]. One other study as well indicated that the embryo implantation rate, clinical pregnancy rate and live birth rate in the recryopreservation group were significantly lower, and the miscarriage rate also slightly increased[11]. In their study, embryos underwent twice cryopreservation in the blastocyst stage, in addition embryo selection was restrictive compared with once cryopreservation group. Moreover, the blastocyst underwent twice frozen- 
thawed, and the times of laser drilling and shrinkage increased, which may affect the implantation ability of embryos. Although current studies have confirmed the safety of laser manipulation in human embryo cryopreservation, however the cumulative effect is unclear[32, 33]. In our study, we selected embryos underwent twice-cryopreservation, once at the cleavage stage and the second time at the blastocyst stage. Blastocyst culture itself was the selection of embryos, only an embryo with developmental potential can form a blastocyst. And the blastocysts underwent the same number of laser drilling as in the one-freeze group.

More and more children are born depending on ART globally, so the safety of the offspring is more concerned. When compared with fresh embryo transfer,FET is associated with higher risk of LGA and macrosomia in singletons $[5,7,34]$. The maternal and fetal health that experiences repeated freezing should be paid more attention to. Our results showed that no significant differences were observed in pregnancy complication rate, gestational age, fetal birthweight, gender, congenital birth defects rate in the singleton delivery between oncecryopreserved and twice-cryopreserved group. However, the macrosomia rate was $19.56 \%$ in the twicecryopreserved group, when it was $6.35 \%$ in once-cryopreserved group, whether the time of vitrification-warming procedure is positively associated with the fetal birthweight need further study. Birthweight may be related to gender, so the incidence of macrosomia, gestational age, birthweight was further analyzed for boys or girls separately, and no significant differences was observed may due to few samples.

There are several limitations in our study. First, it was a retrospective study conducted in a single center, a larger prospective cohort study was needed to validate our findings. Second, although we used propensity score matching to eliminate the influence of baseline characteristics on FET outcomes as much as possible, it could not eliminate all the bias caused by confounders between the two groups.

In summary, our results presented the pregnancy outcomes and neonatal safety of human refrozen-thawed embryos. Twice vitrification of human embryos at different developmental stages do not affect clinical outcomes and perinatal outcomes. The re-cryopreservation procedure would be a valuable option to increase the cumulative pregnancy rate while preventing embryo waste under full informed consent. However, the maternal and child safety of re-cryopreservation procedure still needs to be confirmed by a long-term multicenter prospective cohort study.

\section{Abbreviations}

FET: frozen-thawed embryo transfer; PSM: propensity score matching; CPR: clinical pregnancy rate; LBR: live birth rate; IVF: in-vitro fertilization; OHSS: ovarian hyperstimulation syndrome; LGA: large for gestational age; BMI: body mass index; FSH: follicle-stimulating hormone; HRT: hormone-replacement therapy; HCG: human chorionic gonadotropin; ICSI: intracytoplasmic sperm injection.

\section{Declarations}

\section{Ethics approval and consent to participate}

The study was approved by the Ethics Committee of the Nanjing Drum Tower Hospital, The Affiliated Hospital to Nanjing University Medical School on 6 May 2021 (reference number 2021-163-01). This study was 
conducted at Reproductive Medicine Center of Nanjing Drum Tower Hospital. All patients signed informed consents regarding ART.

\section{Consent for publication}

Not applicable.

\section{Availability of data and materials}

Please contact author for data requests.

\section{Competing interests}

The authors declare that they have no competing interests.

\section{Funding}

This work was supported by the [Jiangsu Provincial Key Research and Development Program \#1] under Grant [number BE2019601]; and [National Natural Science Foundation of China] under Grant [number 81771537].

\section{Author contributions}

JZ contributed to the study design. MD, JX collected data and conducted analysis. YY contributed analysis tools. SW helped to perform the laboratory operations. XS was a major contributor in writing the manuscript. All authors read and approved the final manuscript.

\section{Acknowledgements}

We thank the clinicians, clinical embryologists, laboratory technologists and nurses of the Centre of Reproductive Medicine for data collection.

\section{References}

1. Zhang W, Xiao X, Zhang J, Wang W, Wu J, Peng L, et al. Clinical outcomes of frozen embryo versus fresh embryo transfer following in vitro fertilization: a meta-analysis of randomized controlled trials. Arch Gynecol Obstet. 2018;298(2):259-72.

2. Zhang X, Ma C, Wu Z, Tao L, Li R, Liu P, et al. Frozen-Thawed Embryo Transfer Cycles Have a Lower Incidence of Ectopic Pregnancy Compared With Fresh Embryo Transfer Cycles. Reprod Sci. 2018;25(9):1431-5.

3. Liu X, Bai H, Shi W, Shi J. Frozen-thawed embryo transfer is better than fresh embryo transfer in $\mathrm{GnRH}$ antagonist cycle in women with 3-10 oocytes retrieved: a retrospective cohort study. Arch Gynecol Obstet. 2019;300(6):1791-6.

4. Wong KM, Mastenbroek S, Repping S. Cryopreservation of human embryos and its contribution to in vitro fertilization success rates. Fertil Steril. 2014;102(1):19-26.

5. Smith A, Tilling K, Lawlor DA, Nelson SM. Live birth rates and perinatal outcomes when all embryos are frozen compared with conventional fresh and frozen embryo transfer: a cohort study of 337,148 in vitro 
fertilisation cycles. BMC Med. 2019;17(1):202.

6. Maheshwari A, Pandey S, Amalraj Raja E, Shetty A, Hamilton M, Bhattacharya S. Is frozen embryo transfer better for mothers and babies? Can cumulative meta-analysis provide a definitive answer? Hum Reprod Update. 2018;24(1):35-58.

7. Litzky JF, Boulet SL, Esfandiari N, Zhang Y, Kissin DM, Theiler RN, et al. Effect of frozen/thawed embryo transfer on birthweight, macrosomia, and low birthweight rates in US singleton infants. Am J Obstet Gynecol. 2018;218(4):433 e1- e10.

8. Cutting R. Single embryo transfer for all. Best Pract Res Clin Obstet Gynaecol. 2018;53:30-7.

9. Koch J, Costello MF, Chapman MG, Kilani S. Twice-frozen embryos are no detriment to pregnancy success: a retrospective comparative study. Fertil Steril. 2011;96(1):58-62.

10. Zheng X, Chen Y, Yan J, Wu Y, Zhuang X, Lin S, et al. Effect of repeated cryopreservation on human embryo developmental potential. Reprod Biomed Online. 2017;35(6):627-32.

11. Wang M, Jiang J, Xi Q, Li D, Ren X, Li Z, et al. Repeated cryopreservation process impairs embryo implantation potential but does not affect neonatal outcomes. Reprod Biomed Online. 2021;42(1):75-82.

12. Gardner DK, Schoolcraft WB. Culture and transfer of human blastocysts. Curr Opin Obstet Gynecol. 1999;11(3):307-11.

13. Papanikolaou EG, Camus M, Kolibianakis EM, Van Landuyt L, Van Steirteghem A, Devroey P. In vitro fertilization with single blastocyst-stage versus single cleavage-stage embryos. N Engl J Med. 2006;354(11):1139-46.

14. Papanikolaou EG, Kolibianakis EM, Tournaye H, Venetis CA, Fatemi H, Tarlatzis B, et al. Live birth rates after transfer of equal number of blastocysts or cleavage-stage embryos in IVF. A systematic review and meta-analysis. Hum Reprod. 2008;23(1):91-9.

15. De Croo I, De Sutter P, Tilleman K. A stepwise approach to move from a cleavage-stage to a blastocyststage transfer policy for all patients in the IVF clinic. Hum Reprod Open. 2020;2020(3):hoaa034.

16. Wang N, Zhao X, Ma M, Zhu Q, Wang Y. Effect of Day 3 and Day 5/6 Embryo Quality on the Reproductive Outcomes in the Single Vitrified Embryo Transfer Cycles. Front Endocrinol (Lausanne). 2021;12:641623.

17. Macnamee M, Wick K, Rainsbury P, Sathanandan M, Brinsden P, Edwards RG. How robust are early human embryos? Lancet. 1990;336(8715):636.

18. Baker A, Check JH, Lurie D, Hourani C, Hoover LM. Pregnancy achieved with pronuclear-stage embryos that were cryopreserved and thawed twice: a case report. J Assist Reprod Genet. 1996;13(9):713-5.

19. Yokota Y, Yokota H, Yokota M, Sato S, Araki Y. Birth of healthy twins from in vitro development of human refrozen embryos. Fertil Steril. 2001;76(5):1063-5.

20. Farhat M, Zentner B, Lossos F, Bdolah Y, Holtzer H, Hurwitz A. Successful pregnancy following replacement of embryos previously refrozen at blastocyst stage: case report. Hum Reprod. 2001;16(2):337-9.

21. Smith LK, Roots EH, Dorsett MJ. Live birth of a normal healthy baby after a frozen embryo transfer with blastocysts that were frozen and thawed twice. Fertil Steril. 2005;83(1):198-200.

22. Sills ES, Murray GU, Genton MG, Walsh DJ, Coull GD, Walsh AP. Clinical features and reproductive outcomes for embryos undergoing dual freeze-thaw sequences followed by blastocyst transfer: critique of 14 consecutive cases in IVF. Fertil Steril. 2009;91(4 Suppl):1568-70. 
23. Kumasako Y, Otsu E, Utsunomiya T, Araki Y. The efficacy of the transfer of twice frozen-thawed embryos with the vitrification method. Fertil Steril. 2009;91(2):383-6.

24. Murakami M, Egashira A, Murakami K, Araki Y, Kuramoto T. Perinatal outcome of twice-frozen-thawed embryo transfers: a clinical follow-up study. Fertil Steril. 2011;95(8):2648-50.

25. Farhi J, Elizur S, Yonish M, Seidman DS, Shulman A, Schiff E, et al. Assessment of a double freezing approach in the management of surplus embryos in IVF. Reprod Biomed Online. 2019;38(4):517-9.

26. Bourdon M, Pocate-Cheriet K, Finet de Bantel A, Grzegorczyk-Martin V, Amar Hoffet A, Arbo E, et al. Day 5 versus Day 6 blastocyst transfers: a systematic review and meta-analysis of clinical outcomes. Hum Reprod. 2019;34(10):1948-64.

27. Park DS, Kim JW, Chang EM, Lee WS, Yoon TK, Lyu SW. Obstetric, Neonatal, and Clinical Outcomes of Day 6 vs. Day 5 Vitrified-Warmed Blastocyst Transfers: Retrospective Cohort Study With Propensity Score Matching. Front Endocrinol (Lausanne). 2020;11:499.

28. Goto S, Kadowaki T, Tanaka S, Hashimoto H, Kokeguchi S, Shiotani M. Prediction of pregnancy rate by blastocyst morphological score and age, based on 1,488 single frozen-thawed blastocyst transfer cycles. Fertil Steril. 2011;95(3):948-52.

29. Wang C, Shu J, Lin R, Chen H, Gan X, Deng X, et al. Choosing the optimal blastocyst by morphology score versus developmental rate in frozen-thawed embryo transfer cycles. Hum Fertil (Camb). 2020:1-10.

30. Debrock S, Peeraer K, Fernandez Gallardo E, De Neubourg D, Spiessens C, D'Hooghe TM. Vitrification of cleavage stage day 3 embryos results in higher live birth rates than conventional slow freezing: a RCT. Hum Reprod. 2015;30(8):1820-30.

31. Rienzi L, Gracia C, Maggiulli R, LaBarbera AR, Kaser DJ, Ubaldi FM, et al. Oocyte, embryo and blastocyst cryopreservation in ART: systematic review and meta-analysis comparing slow-freezing versus vitrification to produce evidence for the development of global guidance. Hum Reprod Update. 2017;23(2):139-55.

32. Davidson LM, Liu Y, Griffiths T, Jones C, Coward K. Laser technology in the ART laboratory: a narrative review. Reprod Biomed Online. 2019;38(5):725-39.

33. Aluko A, Vaughan DA, Modest AM, Penzias AS, Hacker MR, Thornton K, et al. Multiple cryopreservationwarming cycles, coupled with blastocyst biopsy, negatively affect IVF outcomes. Reprod Biomed Online. 2021;42(3):572-8.

34. Berntsen S, Pinborg A. Large for gestational age and macrosomia in singletons born after frozen/thawed embryo transfer (FET) in assisted reproductive technology (ART). Birth Defects Res. 2018;110(8):630-43. 